\title{
“Kwe'sx nasa ksxa' wnxi" el gran sueño de los indios: una experiencia de educación indígena y de esperanza de paz en medio del conflicto armado colombiano*
}

\author{
Esperanza Montaño Aedo**
}

Fecha de recepción: 7 de abril de 2015

Fecha de revisión: 16 de mayo de 2015

Fecha de aprobación: 5 de junio de 2015

\section{Resumen}

En el valle del río Cauca en Colombia, Suramérica, existen experiencias pedagógicas rurales que pese a vivir en contextos de violencia y en medio del conflicto armado, sobresalen por su liderazgo, y han sido reconocidas y destacadas como ejemplos de gestión y construcción colectiva. Este artículo hace referencia a la experiencia de la Institución Educativa Indígena IDEBIC, que en lengua Nasa significa "Kwe'sx Nasa Ksxa'wnxi" (El Gran Sueño de los Indios), con la cual se realizó un acercamiento para conocer cómo afecta el conflicto armado que se vive en Colombia, las prácticas pedagógicas, la vida escolar, el trabajo de resistencia cultural y de qué manera los maestros y maestras

* Artículo de reflexión. Este artículo expone algunas reflexiones y resultados preliminares de la investigación: Retos y desafíos de la escuela para la construcción de una cultura de paz. Experiencias pedagógicas significativas en contextos de violencia en el Valle Del Cauca - Colombia. Programa de doctorado en Investigación e Innovación en Diagnóstico y Orientación Educativa. Universidad Nacional de Educación a Distancia - UNED, España, marzo de 2015.

** Supervisora de educación en la Secretaría de Educación departamental. Gobernación del Valle del Cauca. Alumna del doctorado en Investigación e Innovación en Diagnóstico y Orientación Educativa en la UNED de España. Correo electrónico: enredes01@hotmail.com, Dirección de correspondencia: Carrera 10 \# 3-29, barrio San Antonio, Cali,Colombia. 
del IDEBIC han logrado que sus principios de pluriculturalidad y multiculturalidad permanezcan y se fortalezcan a pesar de la presencia en la zona de actores armados.

\title{
Palabras clave
}

Experiencia pedagógica, educación indígena, conflicto armado.

\section{"Kwe'sx nasa ksxa wnxi" the great dream of the indigenous: an experience of indigenous education for hope and peace in the midst of the colombian conflict}

\begin{abstract}
In the valley of the Cauca river in Colombia, South America, we can find pedagogic rural experiences that, despite existing under contexts of armed conflict-related violence, stand out as examples of leadership. Furthermore, these experiences have been recognized as remarkable cases of collective construction and management. This article presents the case of Indigenous Educational Institution IDEBIC (or in Nasa language, "Kwe'sx Nasa Ksxa' wnxi," The Indians Great Dream, with which I conducted research aimed to determine the impact of the armed conflict on pedagogic practices, school life, and cultural resistance work. The article documents the way in which IDEBIC's teachers have been able to maintain and strengthen their multiculturalism and pluralism principles regardless of the presence of local armed actors in the area.
\end{abstract}

\section{Keywords}

Teaching experience, indigenous education, armed conflict. 


\section{"Kwe'sx nasa ksxa "wnxi" o grande sonho dos índios: uma experiência de educação indígena e de esperança de paz no meio do conflito armado colombiano}

\section{Resumo}

No estado chamado Valle del Cauca na Colombia, America do Sul, há experiências pedagógicas rurais que embora estejam num contexto de violência, em meio o conflito armado, destacam pela sua liderança sendo reconhecidas e ressaltadas como exemplo de gestão e construção coletiva.Nesse artigo se faz referência a experiência da Institución Educativa Indígena IDEBIC, que na língua Nasa significa "Kwe'sx Nasa Ksxa' wnxi", o Grande Sonho dos Índios, com ela se fez uma aproximação para conhecer a forma em que o conflito armado afeta as práticas pedagógicas, a vida escolar, o trabalho de resistência cultural e como os professores e professoras do IDEBIC tem conseguido que os seus princípios de pluriculturalidade e multiculturalidade permaneçam e se fortaleçam apesar da presença no território de atores armados.

\section{Palavras-Chave}

Experiência pedagógica, educação indígena, conflito armado. 


\section{Introducción}

La guerra comenzó en el Golfo de Urabá hace quinientos años porque no es de ayer ni de hace veinte o treinta años, desde cuando Juan de la Cosa y Rodrigo Bastidas hicieron su primera masacre en tierra firme contra nosotros los Tules... nuestro tema es cómo la guerra penetró nuestra cultura, pero como estaba fortalecida pudo sobrevivir... la guerra cambia la cultura, el horario y nuestros rituales y costumbres. Las mujeres comenzaron a sentirse muy temerosas y con miedo a ser violadas y entonces los hombres no podían ya ir a trabajar al campo por cuidar a sus mujeres y a sus niños... Entonces comenzamos a fortalecer nuestros rituales, cultura y tradición, a cuidar el territorio, los lugares sagrados, la naturaleza, el agua, los ríos... Hoy el pueblo Tule todavía está vivo y sabemos que sí es posible cuando se tiene conciencia de la dignidad y del respeto a la madre naturaleza (PNUD, 2003).

En Colombia existe una experiencia de educación indígena que ha resistido el embate de la violencia: "Kwe'sx Nasa Ksxa'wnxi", nombre que en lengua Páez significa: El Gran Sueño de los Indios.

Oficialmente creado como el Instituto de Educación Básica Indígena - IDEBIC, está localizado en el departamento del Valle del Cauca, Colombia, Suramérica, y está conformado por 59 escuelas o sedes, localizadas en zonas rurales de este departamento. Su sede principal está ubicada en una zona montañosa del municipio de Florida (Valle), donde hay presencia de varios de los grupos armados de la región.

En el año 2003 se creó el IDEBIC y sus sedes están ubicadas en 18 municipios donde viven comunidades indígenas; estos municipios son: Florida, Bolívar, CalimaDarién, Dagua, El Cairo, El Dovio, Argelia, Jamundí, La Victoria, Pradera, Restrepo, Trujillo, Vijes, Versalles, Ansermanuevo, Bugalagrande, Obando y Yotoco.

EI IDEBIC cuenta con un rector: Aníbal Bubú Ramos, cinco coordinadores y cien docentes que educan a aproximadamente 2.000 estudiantes indígenas.

En su Proyecto Etnoeducativo Comunitario y Complementario - PEC, implementa la educación propia y el aprendizaje de la lengua materna, tanto en la comunidad Nasa Páez como en los Embera Chamí, pues para las comunidades indígenas cada lengua es instrumento del pensamiento y de la comunicación en su grupo étnico, correspondiendo a una concepción del mundo y a un modo de pensar que es transmitido al niño al aprender la lengua materna en sus primeros años. 
La experiencia pedagógica del IDEBIC ha sido reconocida como una de las más significativas del país, destacada en diversos eventos como un ejemplo de gestión y construcción colectiva y de superación de las dificultades que conlleva estar en medio del conflicto armado.

El acercamiento inicial a la experiencia se realizó partir de dos interrogantes:

- ¿Cómo afecta el conflicto armado que se vive en Colombia, las prácticas pedagógicas, la vida escolar y el trabajo de resistencia cultural de la institución de educación básica indígena IDEBIC?

- ¿De qué manera han logrado los maestros y maestras del IDEBIC que sus principios de pluriculturalidad y multiculturalidad permanezcan y se fortalezcan a pesar de la presencia en la zona de actores armados?

\section{Una mirada al contexto}

En el departamento del Valle del Cauca existen 22 resguardos, de los cuales 7 se encuentran en el municipio de Buenaventura, 3 en el municipio de Florida y el resto distribuidos en otros 11 municipios. La población indígena del Valle del Cauca tiene un peso porcentual del $0,87 \%$ en la población total de los municipios con población indígena, y respecto a la nación este número de población ubica al departamento en el lugar 18 de los 28 departamentos con población indígena en el país (Secretaría de Educación, Gobernación del Valle del Cauca, 2008).

Los grupos armados han convertido a los pueblos indígenas en una de sus principales víctimas: entran a sus territorios, desconocen sus autoridades autóctonas, les imponen su ley de sangre y fuego, les desplazan para apropiarse de sus tierras y de sus recursos naturales. La mayoría de los líderes indígenas son desaparecidos o asesinados y se han cometido actos violatorios de los Derechos Humanos y el Derecho Internacional Humanitario como los bloqueos, hostigamientos y cercos, muchos de los cuales no son denunciados por los indígenas por el temor a las retaliaciones.

El informe de Desarrollo Humano dice que:

En el año 2002, en Colombia fueron asesinados 107 indígenas, los desplazamientos forzados afectaron a diez mil, las incursiones de grupos armados en 
territorios ancestrales a quince mil, los daños a la infraestructura de servicios a dos mil, los controles a la circulación de alimentos a tres mil, la contaminación de suelos y aguas a 750 personas (PNUD, 2003).

En los últimos años, estas cifras, en lugar de disminuir, continuaron en aumento pues permanentemente se presentan agresiones contra los pueblos indígenas a pesar de sus marchas y la movilización masiva para denunciar ante la comunidad internacional las violaciones a sus Derechos Humanos y el peligro inminente de exterminio de su cultura.

La historia de las poblaciones indígenas en Colombia, ha sido una historia de exclusión y opresión, de violaciones a sus Derechos Humanos y de luchas para reivindicar sus derechos fundamentales, entre ellos el derecho a una educación propia que les permita enfrentar las consecuencias de la llamada "civilización", léase ahora "modernidad", que trajo consigo la pérdida de sus lenguas, su memoria, sus culturas.

Frente a este panorama, ha ido tomando fuerza el movimiento indígena como uno de los ejemplos de resistencia cultural más fuerte de América Latina. La exigencia de una educación propia a través de la etnoeducación desde la década de 1970, ha resultado en la creación de instituciones educativas adecuadas a las particularidades culturales, centradas en la defensa del territorio y en la diversidad y riqueza cultural de las diferentes etnias.

La Corte Constitucional en una de sus sentencias expresó:

[...] El conflicto armado colombiano amenaza con el exterminio cultural o físico a numerosos pueblos indígenas del país. En el curso de la última década, el conflicto armado que se desarrolla en Colombia, reorientado por actividades relacionadas con el narcotráfico, se ha convertido en el principal factor de riesgo para la existencia misma de docenas de comunidades y pueblos indígenas a lo largo del territorio nacional. (Protección de los derechos fundamentales de las personas y los pueblos indígenas desplazados por el conflicto armado Auto 004, 2009). 


\section{Educación propia e identidad cultural}

Colombia no ha sido ajena al "asimilacionismo cultural" que ha vivido América Latina, relacionado con el papel que cumplen los sistemas oficiales de la educación pública en el proceso de asimilación de lo indígena a una "cultura nacional", a pesar de la referencia a un estado pluricultural y multiétnico en la Constitución Nacional.

El afán de homogenizar de la educación formal a través de su currículo oficial, sus estándares y competencias y en general, a través de las políticas públicas, ha permeado la educación en las comunidades indígenas que se debaten entre una educación "propia" que rescate su identidad y revalore su cosmovisión, y las exigencias de un sistema que los clasifica en "superiores, inferiores o muy inferiores" según los resultados obtenidos en las pruebas de conocimientos de los estudiantes.

[...] El asimilacionismo cultural es la política que se ha procurado sostener desde el Estado, a través del sistema institucionalizado de educación pública. La estrategia ha consistido y consiste en una 'asimilación' de los 'indios' en la cultura de los dominadores, que suele ser también mentada como la 'cultura nacional', a través de la educación escolar formal, sobre todo, pero también por el trabajo de instituciones religiosas y militares (Quijano, 2005).

La educación oficial ha sido un mecanismo de "des-indianización subjetiva, cultural" combinada con una política de discriminación y de enajenación de lo 'indio'. Aquí des-indianización se refiere a la destrucción, y apropiación de las conquistas culturales indígenas: Según Quijano (2005): “la colonialidad del poder sigue implicando que toda o parte de las poblaciones no 'blancas' no pueden consolidarse en su ciudadanía sin originar profundos y graves conflictos sociales".

El proyecto intercultural en el discurso de los movimientos indígenas está proponiendo una transformación. No están pidiendo el reconocimiento y la inclusión en un Estado que reproduce la ideología neoliberal y el colonialismo interno, sino que están reclamando la necesidad de que el Estado reconozca la diferencia colonial (ética, política y epistémica). Tampoco están pidiendo que se reconozca la participación de los indígenas en el Estado, sino la intervención en paridad y reconociendo la diferencia actual de poder, esto es, la diferencia colonial y la colonialidad del poder todavía existente, de los indígenas en la transformación del Estado, y por cierto, de la educación, la economía, la ley (Walsh, 2003). 


\section{Las comunidades indígenas y el conflicto armado}

El conflicto armado interno que sufre Colombia tiene profundas raíces sociales, económicas y culturales. La población civil sufre los mayores efectos de este conflicto, porque las partes en contienda continúan cometiendo graves infracciones al derecho humanitario. Las estadísticas señalan que en promedio, en los últimos años, de cada cuatro personas que mueren por causa del conflicto, tres son civiles.

En el año 2003, la Alta Comisionada de las Naciones Unidas para los Derechos Humanos, señaló que la situación de derechos humanos en Colombia se ha caracterizado por "violaciones graves, masivas y sistemáticas de esos derechos, constituyendo muchas de ellas crímenes contra la humanidad" (ONU, 2003).

Cinco años después, "las violaciones continúan siendo graves, masivas y sistemáticas, en muchos casos se han agudizado, y en gran parte permanecen en la impunidad..." (Rey, 2008).

Las comunidades indígenas son algunas de las más afectadas por este conflicto: en el año 2004, el informe Stavenhagen en el Foro Permanente de Naciones Unidas para las Cuestiones Indígenas, reveló que numerosas comunidades indígenas denuncian asesinatos selectivos de sus líderes y voceros y de sus autoridades tradicionales. Estos homicidios, que parecen formar parte de estrategias diseñadas a descabezar y desorganizar a las comunidades indígenas, contribuyen a la desintegración social y cultural de las mismas. "Se trata de verdaderos genocidios y etnocidios perpetrados contra los pueblos indígenas" (Stavenhagen, 204).

En el año 2009, el informe del Asesor Especial para la Prevención del Genocidio, planteó que en Colombia hay comunidades indígenas que se encuentran amenazadas con el exterminio cultural o físico, no necesariamente cruenta e igualmente así genocida. Esto con base en la Convención para la Prevención y Sanción del Delito de Genocidio y en el Estatuto de la Corte Penal Internacional.

Una de las recomendaciones del informe sobre la situación de los pueblos indígenas en Colombia del Relator Especial de las Naciones Unidas sobre la situación de los derechos humanos y las libertades fundamentales de los indígenas, dice:

Se urge al Estado a invitar al Asesor Especial para la Prevención del Genocidio de las Naciones Unidas para monitorear la situación de las comunidades indígenas que se encuentran amenazadas con el exterminio cultural o físico, según la Corte Constitucional en su Auto 004 de 2009. Así mismo, insta al Estado a continuar su cooperación con el Fiscal de la Corte Penal Internacional (Anaya, 2010). 
El periódico digital "Actualidad Étnica" realizado por la Fundación Hemera Comunicar, publicó en noviembre de 1999:

[...] El conflicto interno armado en Colombia no solo afecta a miles de personas que viven en el campo o en la ciudad, sino que ha llegado a territorios recónditos, para algunos, tierra sagrada, pero para otros tierra para explotar. El territorio indígena pasó de ser el lugar en donde se converge con la madre naturaleza, en donde se vive con ella y para ella, a ser otro sitio más de lucha y guerra, muerte, sangre y sufrimiento. Para los indígenas, la guerra en Colombia es ajena a nuestro pensamiento y a nuestras actuaciones sociales y políticas, en la cual nosotros somos víctimas de una política de exterminio y aniquilamiento de características etnocidas, dado el impacto negativo sobre la vida y cultura de las comunidades y pueblos indígenas (Vera, 2003).

De acuerdo al análisis de las diferentes organizaciones indígenas, la principal causa del conflicto está relacionada con sus territorios, puesto que "representan corredores geo estratégicos de transito de los grupos armados ilegales y zonas de repliegue o posicionamiento para el desarrollo de sus actividades". Además, en los territorios indígenas hay recursos naturales de gran valor económico como: agua, madera, oro, petróleo, carbón y otros minerales cuya posibilidad de explotación los convierte en zonas de alto valor estratégico.

[...] Los territorios indígenas son considerados potencial para la inyección de grandes capitales, ya sea mediante la explotación de los recursos naturales y el desarrollo de grandes obras de infraestructura... en los campos energético, hídrico y de comunicaciones; o mediante la creación de industrias ilícitas ligadas al narcotráfico, la guerra, el tráfico de armas y el secuestro (Ibídem).

El conflicto armado ha causado el desplazamiento de comunidades indígenas, quienes han tenido que huir de su territorio por temor a las amenazas de los actores armados.

Al respecto, la autora plantea que:

[..] El desplazamiento es no solo el despojo de tierras, sino también una táctica de desarticulación de las comunidades, lo cual genera la descomposición de su tejido social... este desarraigo no solo impacta negativamente la vida de quienes 
se desplazan; también afecta a los miembros de la comunidad que se quedan: la zozobra, el temor y las limitaciones afectan su vida material y económica, emotiva y espiritual, su identidad cultural, su ejercicio de la autoridad, su capacidad de gobierno, sus procesos de organización, su capacidad de administrar la justicia propia y de ejercer la autonomía (Ibídem).

El desplazamiento forzado se constituye hoy en día por su magnitud y características, en una verdadera crisis humanitaria y una grave violación a los derechos humanos, civiles y políticos convirtiéndose este grupo de población en el grupo más vulnerable, no solo por las carencias materiales que afronta al huir de su lugar de origen sino por el efecto psicosocial que tiene el desarraigo en su capacidad de agenciar su propio proyecto de vida.

Según el Informe del Relator Especial de la ONU, James Anaya, donde presenta las observaciones sobre la situación de los derechos humanos y las libertades fundamentales de los indígenas en Colombia, a pesar de algunos avances en materia indígena, en general, las leyes, programas y políticas del Gobierno no permiten una efectiva protección y satisfacción de los derechos humanos de los pueblos indígenas en el país:

[...] Es evidente que la situación de los indígenas resulta exacerbada e intensificada por causa del conflicto armado interno que aflige al país. La precaria situación de los derechos humanos de los pueblos indígenas en Colombia refleja el desfase entre una legislación nacional avanzada y la poca efectividad real de las instituciones encargadas de su protección, en el marco de un conflicto armado interno en el cual participan numerosos contendientes cuya acción incide en las posibilidades de supervivencia de las comunidades autóctonas (Anaya, 2010).

En Colombia se ha avanzado en el reconocimiento constitucional y legal de los derechos humanos de los pueblos indígenas. Pero el conflicto armado interno, las bandas criminales y el narcotráfico, no han posibilitado dicho reconocimiento, por lo que es indispensable que el Estado proteja los derechos de los pueblos indígenas y aplique los mecanismos necesarios para garantizarlos. 


\section{La escuela en medio del conflicto}

La escuela indígena no ha estado exenta de la violencia que genera el conflicto armado en Colombia, y para resistir ha tenido que innovar tanto sus prácticas pedagógicas como sus procesos de gestión académica, administrativa y comunitaria.

La coalición contra la vinculación de niños, niñas y jóvenes al conflicto armado en Colombia conformada por diferentes organizaciones civiles que luchan para proteger a los niños y jóvenes, hizo los siguientes planteamientos que ilustran lo que ha venido ocurriendo en las escuelas como consecuencia del conflicto armado (Fundación Dos Mundos, 2003):

1. La escuela es uno de los espacios más afectados por el conflicto armado, y paralelamente el derecho a la educación de niños y niñas se ha visto seriamente vulnerado en razón del conflicto.

2. La escuela se ha visto afectada por la presencia de grupos armados en la zona, con sus consecuentes limitaciones de la libertad de desplazamiento, mecanismos de control social y constantes hechos violentos, dificultando la asistencia de los niños a la escuela y generando en muchos casos estrés, depresión e incapacidad para concentrarse en las actividades académicas.

3. Las escuelas, alumnos y maestros son constantemente blanco de ataques, lo que ha llevado en algunos casos, a la decisión de interrumpir la prestación del servicio por los problemas de orden público de la zona.

Según el periódico El Tiempo en su publicación del 28 de febrero de 2003: En el departamento del Cesar los indígenas kankuamos denunciaron que el ELN asesinó a dos estudiantes del colegio San Isidro Labrador por negarse a unirse a sus filas. Los indígenas señalaron que de 500 estudiantes que tenía el colegio en el 2002, solamente quedaron 300 en el 2003 (Ibídem).

La desescolarización, vinculada al desplazamiento forzado de niños y niñas, al cierre de centros educativos o simplemente al miedo, puede favorecer graves vulneraciones a sus derechos, como la explotación sexual, prostitución infantil o la vinculación a un grupo armado. 
Los actores armados hacen uso de las escuelas de diferentes maneras, poniendo en riesgo tanto las vidas de los y las estudiantes, como las instalaciones educativas:

En el marco de la operación Mariscal, realizada conjuntamente por la Policía, Fiscalía, DAS, Cuarta Brigada del Ejército y la Fuerza Aérea en mayo del 2002, las fuerzas armadas estatales usaron como escudo el edificio del Liceo Barrio la Independencia, de manera que la escuela estuvo durante 8 horas en medio de la confrontación. Publicado en El Tiempo, 23 de mayo de 2002 (Ibídem).

\section{EI IDEBIC: una esperanza de paz}

Desde hace 12 años se ha venido construyendo la experiencia pedagógica de los maestros indígenas del IDEBIC, en un proceso de resistencia cultural frente a los riesgos de exterminación de sus comunidades étnicas y a la pérdida de su identidad ocasionada por el afán colonizador que aún persiste en América Latina.

En 1998 se fundó la Institución Educativa, que en aquel entonces se llamaba el Instituto Departamental de Educación Básica Comunitaria y Complementaria IDEBIC. Con ese nombre nació el 18 de octubre de 1998. Ahí abrimos el nivel de bachillerato, iniciamos con sexto y fue un proceso muy duro, nacido desde la marginalidad, desde la ignorancia por parte del Gobierno porque nos ignoraba totalmente, desde la estigmatización, la marginación (Ramos, 2010).

En la Institución Educativa Indígena IDEBIC en Florida, Valle del Cauca, se desarrolla una experiencia pedagógica fundamentada en los principios de la etnoeducación.

Iniciamos con todas las dificultades del caso, con un colegio sin reconocimiento legal, estuvimos sin este reconocimiento desde 1998 hasta el 2003, casi 5 años sin reconocimiento, legal para nosotros, ilegal para el gobierno. La gente decía que ese colegio era un colegio "pirata" porque no era reconocido y con la comunidad indígena se hizo un proceso de movilizaciones, de marchas, pero es a partir del 28 de diciembre del 2003, que se le da ese reconocimiento gracias 
al decreto 712 y ya de ahí, se le da toda la potestad de cumplir con la educación en todos los niveles, de la básica, secundaria y media, y hoy ya estamos es "a par" (Ibídem).

El currículo que se ha venido construyendo en el IDEBIC recupera su dimensión social y cultural al incluir en el plan de estudios contenidos orientados a preservar la cosmovisión y la cultura Nasa Páez.

Los mayores pensaban en la educación como una estrategia de supervivencia cultural, porque antes la educación que había sido homogénea para el pueblo colombiano, no tenía en cuenta las particularidades o singularidades de los pueblos indígenas, entonces ese tipo de educación que se empezó a construir y a implementar en la estrategia educativa, quiere impulsar los principios de las organizaciones indígenas, como son la unidad, la tierra, la cultura, la autonomía. Los profesores están en una dinámica donde enseñan dignidad indígena, lengua materna, legislación, cosmovisión, filosofía propia, derecho mayor, ley de origen, medicina propia (Ibídem).

El conflicto armado en medio del cual se desarrolla esta experiencia de resistencia cultural y social, afecta profundamente la vida en las comunidades indígenas y en la institución educativa, hasta el punto de que las Naciones Unidas denomina "etnocidio" lo que sucede con varias comunidades indígenas en Colombia. Resistirse a las exigencias de los grupos armados por involucrarse en una guerra ajena para los indígenas, ha sido la principal estrategia de supervivencia de esta comunidad. Pero el miedo y la constante zozobra hacen parte de la vida cotidiana escolar.

Florida es un sitio muy golpeado, han sido 30 años muy duros donde se libra una guerra de los actores armados, llámense militares, guerrillas, paramilitares, en la cual nos quieren involucrar, pero las comunidades indígenas no hemos querido ser partícipes. Sin embargo a través de engaños, de mentiras, porque los grupos armados quieren verse como muy buenos, engañan a los jóvenes y se los van Ilevando, reclutándolos. Nosotros con este proyecto de educación desde que iniciamos, hemos frenado todo eso, porque se les está alertando a los jóvenes que el estudio es para servir a la comunidad, para servir a los cabildos, para servir a su organización propia, para defender su pueblo, su familia, pero no con armas, 
sino con sabiduría, con ideas, con pensamientos y eso es lo que se le enseña a los muchachos (Ibídem).

La riqueza de los territorios indígenas es una de las causas principales del interés de los grupos armados quienes se disputan el poder sobre el territorio, dado el valor estratégico tanto económico como político y social de estas tierras que poseen una variedad natural y geográfica de gran riqueza.

... Todo es por una pelea territorial, una pelea de poderes, de los actores armados que pelean en una tierra que no es de ellos, hay muchos intereses por los recursos naturales, el agua, la biodiversidad, las minas que están dentro del territorio y eso suscita toda esa guerra... (Ibídem).

La educación en la experiencia del IDEBIC se concibe como un espacio de reconstrucción de la memoria e identidad indígena en medio del conflicto. Surge a partir de aquí las siguientes preguntas: ¿cómo se reconstruye esta memoria de lo indígena? ¿La educación plantea un vínculo con otros momentos del conflicto y el papel de los pueblos indígenas en ellos? ¿Cómo convertir en un reto de la educación, hacer un ejercicio de recuperación de la memoria e identidad indígena que incorpore luchas previas o momentos previos del conflicto? Por ejemplo, ¿cómo se asume la identidad del indígena luchando por la tierra como la experiencia de Quintín Lame y como parte de la memoria e identidad indígena?

... El $60 \%$ de la superficie del municipio es territorio indígena, el $40 \%$ de la tierra es de los otros compañeros campesinos y del resto de los ciudadanos del municipio. La diferencia que hay es que aquí en Florida son 82.000 habitantes, nosotros tenemos el $60 \%$ y somos 5.600 apenas, entonces estamos hablando de que más o menos 73.000 personas viven en el $40 \%$, y ese $40 \%$ ni siquiera es de los campesinos, ese $40 \%$ corresponde a lo que es cultivos de caña, a los grandes cultivadores de caña. La tierra está en manos de los cañeros acá en la parte baja y en la parte alta de los asentamientos indígenas. Hemos tenido mucho problema porque los cañeros han tenido la intención de apoderarse de las aguas porque necesitan el agua para sus riegos y el $100 \%$ de las aguas está en territorio indígena... (Ibídem). 
El conflicto, es, entre otros, un espacio de producción de identidades. Dentro del conflicto se es "en contra de" o "a favor de". La educación es por excelencia un lugar de construcción y constante re-creación de la identidad: se es parte de una comunidad. ¿Cómo la escuela es un lugar donde se negocian tanto las identidades del grupo como las que genera el conflicto?

Algo que nosotros hemos logrado rescatar es que hoy un jovencito indígena diga, "oiga, yo soy Nasa" porque antes decía: "no, no yo no sé qué soy, no sé si seré", entonces para nosotros eso es muy valioso porque el dirá: "yo no sabía que soy indígena y que gracias a nuestros ancestros tenemos un origen propio y por lo menos nuestra cultura tiene muchos elementos, tiene una lengua, tiene una música propia y conocimientos muy importantes (Ibídem).

El conflicto implica una redefinición de nación: nuevas concepciones de lealtad, patriotismo, ciudadanía; también implica la disputa de territorios, fronteras interiores y pertenencias. La pregunta que surge es cómo los indígenas se ubican en relación con estas definiciones. Si el multiculturalismo quedó plasmado en la Constitución de 1991, ¿dónde se ubican con respecto al actual proceso de paz? ¿Cuál es el papel de la educación indígena en este sentido?

El proyecto educativo indígena está ligado a la relación de sus comunidades con la tierra. Siendo la tierra un elemento en disputa en el conflicto, icómo se han reacomodado las concepciones indígenas sobre la tierra y qué papel ha tenido en esto la educación? Si los actores armados disputan el territorio, ¿cómo abordan los indígenas esta disputa y los desplazamientos forzados? Si las demandas por la tierra continúan siendo centrales en el conflicto, ¿qué posición toman los indígenas frente a esas demandas? ¿Cómo incorporan la idea de la tierra y el territorio en su proyecto educativo?

Se resalta la incorporación del trabajo de la tierra dentro de las actividades de formación y cómo la escuela se convierte en un lugar que provee no solo instrucción formal sino medios inmediatos para la subsistencia. En ese sentido, la escuela también aparece como un lugar de interrupción del conflicto, en la medida en que se recupera la normalidad y continuidad del trabajo con la tierra (elemento central de la identidad indígena).

Tenemos proyectos económicos y proyectos alimenticios. En lo alimenticio tenemos la huerta, donde se cultiva lechuga, acelga, cilantro, cimarrón, tomillo, 
orégano, zanahoria, cebolla. También se cultiva banano en buena cantidad y es para el consumo y para la venta. Se cultiva café, trigo, maíz, yuca, arracacha y también se tiene ganado y especies menores como gallinas, bimbos, marranos, pollos.

La jornada empieza en que cada profesor acompaña a un grado, es decir, coge un grupo de alumnos a trabajar. Puede que un día sea para recoger el café o desyerbar. Hoy por ejemplo están desyerbando porque la cosecha ya pasó, están haciendo mantenimiento. Es un espacio de 50 minutos que está en el horario, pero en realidad es poco tiempo porque los estudiantes ocupan 10 minutos cogiendo la herramienta, trabajan en la tierra y luego vuelven a dejar la herramienta. Cuando hay que coger café o el trabajo es muy grande, entonces vienen los papas. Los papas se ponen de acuerdo y vienen puntualmente a colaborar en la finca (Ibídem).

Surge como un aspecto a profundizar, el de la escuela como lugar de construcción de comunidad en medio del conflicto. La educación como lugar que reproduce el privilegio o como reproducción de estructuras de poder. A pesar de hablar de la necesidad de tener un proyecto propio como respuesta a esas estructuras de poder y desigualdad, al mismo tiempo con la escuela se continúa la idea de la meritocracia y la excelencia propia de la educación: a los estudiantes se les prepara para tener puntajes altos en pruebas estandarizadas.

En ese sentido, la escuela cumple un cometido pero, iqué se plantea sobre la persistencia de las estructuras de poder y desigualdad? ¿Hay alguna crítica interna sobre el papel social de la escuela?

Nosotros creíamos que no éramos nada cuando comenzamos y llegó un momento por allá en el año 2000 a 2002 que éramos categoría baja, pero luego aparecimos en categoría media y luego subimos a categoría alta. Ahí estuvimos 2 años, luego en el año 2009 presentamos 25 jovencitos al ICFES, y de los 25 todos entraron entre 570.000 estudiantes que se presentaron al ICFES en Colombia. Estos jóvenes indígenas salieron entre los 761 mejores, estamos en el ranking digamos de los 1.000 mejores a nivel de Colombia. De esos 25, 5 entraron entre los 100 mejores... (Ibídem). 
En junio de 2013 la Fundación Compartir otorgó el premio al mejor rector de Colombia a Aníbal Bubú Ramos², rector del Instituto Departamental de Educación Básica Indígena Comunitaria (IDEBIC) del Valle del Cauca, escogido entre 480 rectores postulados de todo el país, por su liderazgo desde hace 10 años en la institución que cuenta con 59 sedes rurales ubicadas en 18 municipios del Valle del Cauca, en las cuales se brinda educación básica primaria, secundaria y media a 2.100 estudiantes indígenas de las etnias Nasa y Embera Chamí.

Los alumnos del IDEBIC estudian materias como: cosmovisión indígena, lengua materna, ética y valores propios de la comunidad indígena para que la cultura de los mayores no se pierda, articulando los conocimientos y conceptos de la cultura occidental con los de la cultura indígena propia. En los últimos cinco años, el IDEBIC ha estado entre las mejores instituciones públicas del departamento, en categorías alta y superior, y ha tenido 15 alumnos entre los mejores estudiantes de todo el país.

El proyecto 'Educando en la diversidad', parte de la complejidad de educar a niños indígenas de la etnia Nasa y de la etnia Embera Chamí en sus respectivas costumbres y cosmovisiones con un enfoque agroambiental y cultural, pues los pueblos indígenas son de vocación agrícola y tienen un estrecha relación con la naturaleza. También forma a los estudiantes en artesanía, música y danza indígenas y en el fortalecimiento de su propia lengua.

Hoy, frente a la expectativa de un acuerdo de paz en el marco de los diálogos que se llevan a cabo en La Habana-Cuba, miramos con ilusión la experiencia del IDEBIC, seguros de que, desde la escuela, se construyen día a día los cimientos para una paz verdadera, con equidad y justicia social.

\section{Referencias Bibliográficas}

Anaya, J. S. (2010, enero). La situación de los pueblos indígenas en Colombia. Naciones Unidas, Asamblea General. A/HRC/15/34/. Disponible en http://www.hchr.org.co/documentoseinformes/documentos/relatoresespeciales/2010/InformeRelatorPueblosIndigenas.pdf

1 Entrevista realizada el día 18 de junio de 2010 en la casa Indígena de Florida, Valle del Cauca, Colombia. 
Calvero, B. (2010). Alerta por Genocidio en Colombia. Foro Permanente de Naciones Unidas para las cuestiones indígenas. Revista Actualidad Étnica. Disponible en http:// Actualidad.Hemeracomunicar.Org/

El Conflicto Callejón con Salida. (2003). Informe Nacional de Desarrollo Humano Colombia. Programa de las Naciones Unidas para el desarrollo PNUD.

Educación y Diversidad Cultural. (2008). Lecciones desde la práctica innovadora en América Latina. Santiago de Chile: Unesco.

Fundación Dos Mundos. (2003). Niñez, escuela y conflicto armado en Colombia. Coalición contra la vinculación de niños, niñas y jóvenes al conflicto armado en Colombia. Bogotá. Disponible en http://www.coalico.org/archivo/InformeCNE_oct.doc . (2003, febrero). Informe de la Alta Comisión de las Naciones Unidas para los Derechos Humanos sobre la situación en Colombia. Doc. E/ CN.4/2003/13, p. 5.

Jumi, G. (2004). Verdad y reparación, derechos mínimos para los pueblos indígenas víctimas del conflicto interno y condiciones esenciales en los procesos de paz. Revista Actualidad Étnica. Disponible en http://actualidad.hemeracomunicar.org/

Quijano, A. (2005, enero-abril). El Movimiento indígena y las cuestiones pendientes en América Latina. Revista Tareas, 119, 31-62. Panamá: CELA, Centro de Estudios Latinoamericanos, Justo Arosemena. Disponible en http://bibliotecavirtual.clacso.org.ar/ ar/libros/tar119/quijano.rtf

. (2008, diciembre). Plan sectorial de educación 2008-2011 Gobernación del Valle del Cauca. Secretaria de Educación.

Ramírez, Á. M. (2001). Paradigma de la interculturalidad. Boletín Instituto Científico de Culturas Indígenas ICCI-ARY Rimay, año 3, No. 26.

Vera, A. J. (2003). Impacto del conflicto armado en Colombia en los territorios Indígenas. Revista Actualidad Étnica. Disponible en http://actualidad.hemeracomunicar.org/

Walsh C. (2003). Las Geopolíticas del conocimiento y la colonialidad del poder. Entrevista a Walter Mignolo. Polis Revista Académica Universidad Bolivariana de Chile, revista online, 1(4). Disponible en http://www.revistapolis.cl/4/wal.pdf

Walsh, C. (2004). Geopolíticas del conocimiento, interculturalidad y descolonialización. Ponencia presentada en la inauguración de la casa de ICCI. Boletín Instituto Científico de Culturas Indígenas ICCI-ARY Rimay, año 6, No. 60. Disponible en http://icci.nativeweb.org/boletin/60/walsh.html 